\title{
Startup and Commissioning Procedures for Electronically Line-Shafted Paper Machine Drives
}

\author{
M. Aníbal Valenzuela and Robert D. Lorenz, Fellow, IEEE
}

\begin{abstract}
Electronic line-shafting control has demonstrated very promising features when applied to paper machine drives. To apply this new control methodology, it is necessary to develop a starting procedure for each individual section and a method for setting the parameters of the virtual line-shaft drive and in-shafts. This paper proposes and evaluates startup and commissioning procedures for electronically line-shafted paper machine drives. The procedure for acceleration of the individual sections is implemented using virtual variable-ratio gearboxes that emulate the physical clutches and conical pulleys used in the era of line-shafts. System stiffness is implemented using virtual in-shafts with active damping. Evaluation shows that the proposed virtual clutch/conical pulley assembly and active damping allow smooth acceleration of each section and maintain well-behaved response during load disturbances for all operating conditions, even with different numbers of sections effectively connected.
\end{abstract}

Index Terms-Active damping, active differential damping, differential motion control, electronic line-shafting control, paper machine drives, synchronized motion control, virtual line-shafts.

\section{INTRODUCTION}

$\mathbf{C}$ URRENT synchronized motion control methods used in paper machines do not possess the intershaft stiffness properties of classical line-shaft mechanically coupled paper machine drives and, consequently, cannot maintain intershaft coordination during transient or variable operating conditions.

In [1], an "electronic line-shafting" control technique was presented based on extension of earlier methods in [2]. Electronic line-shafting was shown to emulate the desirable properties of the physical line-shaft drive. In this control, each sectional drive is torque controlled. Position and velocity outputs of the virtual line-shaft feed the virtual in-shaft of the section producing the torque reference to the drive. This torque is also reflected back to the virtual line-shaft drive as load torque feedback, producing a robust "physical" synchronization between the virtual reference and the follower drives. The method was evaluated on a five-axes web machine for precision filament winding.

Paper PID 02-09, presented at the 2001 IEEE Pulp and Paper Industry Conference, Atlanta, GA, June 19-23, and approved for publication in the IEEE TRANSACTIONS ON INDUSTRY APPLICATIONS by the Pulp and Paper Industry Committee of the IEEE Industry Applications Society. Manuscript submitted for review June 30, 2001 and released for publication March 25, 2002.

M. A. Valenzuela is with the Department of Electrical Engineering, University of Concepción, Concepción, Chile (e-mail: avalenz@die.udec.cl).

R. D. Lorenz is with the Departments of Mechanical Engineering and Electrical and Computer Engineering, University of Wisconsin, Madison, WI 53706-1572 USA (e-mail: lorenz@engr.wisc.edu).

Publisher Item Identifier 10.1109/TIA.2002.800566.
In [3], the application of this new control technique to paper machine drives was presented. Attention was focused on the capability of this control to properly handle sustained torque and/or velocity saturation of any sectional drive. Experimental evaluation in a four-section setup showed well-behaved performance and coordinated motion, both under severe load disturbances and drive saturation.

To apply electronic line-shafting control to paper machine drives, the starting procedure of each individual section and the selection of the virtual shaft parameters need to be addressed. This paper proposes and evaluates such procedures both by simulation and experimentally in a four-section laboratory testbed.

\section{Starting Procedure For Sections}

In the physical line-shaft drives (see Fig. 1), the starting procedure has two steps. First, the line-shaft drive (and the attached shaft) is accelerated with all the mechanical sections unclutched. Then, one by one, each section is accelerated up to the line-shaft speed by closing the section clutches. During the acceleration of a section, the speed of the line-shaft—and, therefore, of all the other sections already connected-will decrease somewhat as some of the stored kinetic energy is effectively transferred to the section being started.

The proposed section starting procedure for the electronic line-shafting control again emulates the desirable properties of the physical line-shaft and improves its deficiencies. This is done by creating a virtual variable-ratio clutch/conical pulley assembly which emulates the clutch and conical pulleys of the physical system (Fig. 2).

To smoothly accelerate the sections, the virtual clutch assembly generates smooth S-shaped velocity ramps (constant positive jerk, constant acceleration, constant negative jerk) with the elapsed time of the ramp set by the operator. Fig. 3 shows the S-ramp and the different segments defined in it. As can be seen, this perfectly shaped S-ramp will have superior performance compared to that the physical clutch, assuring smooth acceleration of the sections and avoiding oscillation in the system components. The starting block works in combination with the draw signal, allowing the operator to increase/decrease the section speed as needed.

\section{SELECtion of System PARAmeters}

\section{A. Virtual Line-Shaft Parameters}

In the physical system, the line-shaft motor rating is selected according to the operating and starting requirements of the dif- 


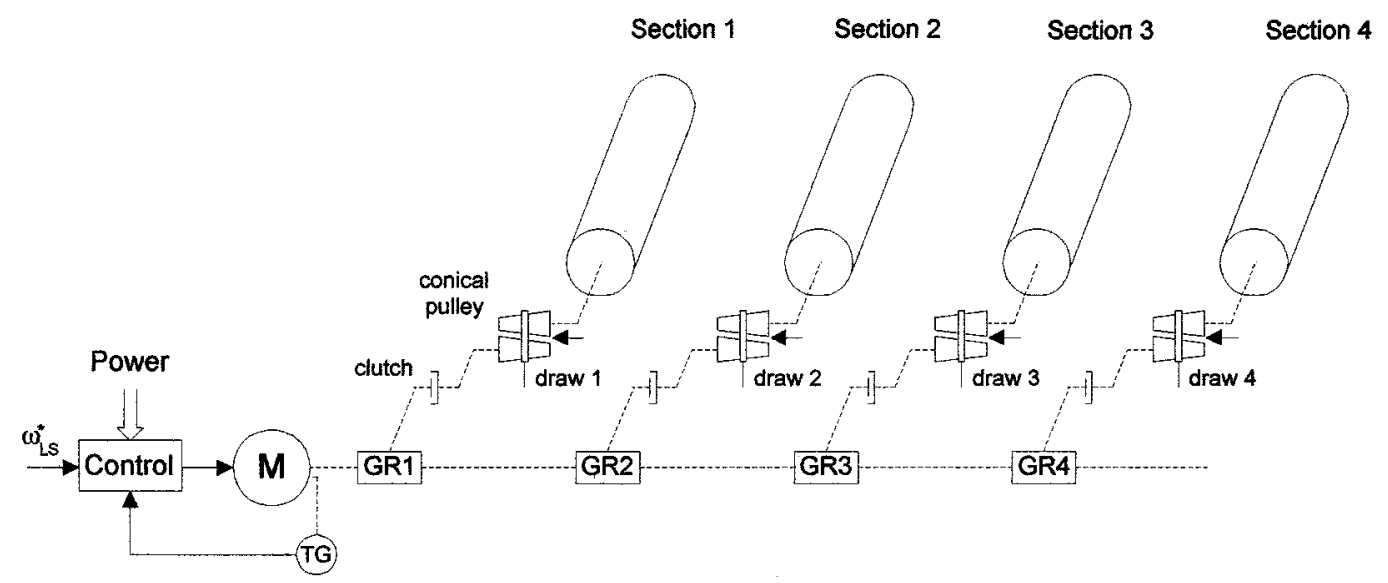

Fig. 1. Line-shafted drive including clutches for accelerating the sections.

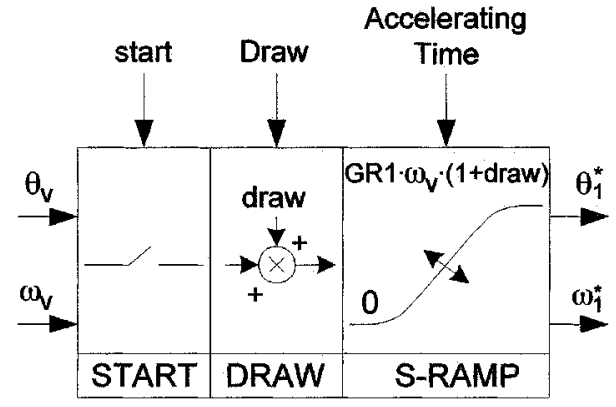

Fig. 2. Virtual variable-ratio clutch/conical pulleys assembly.

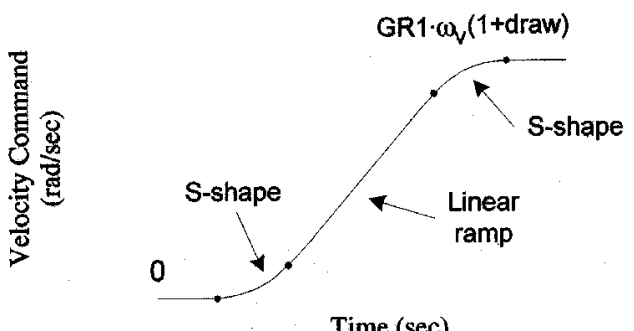

Time (sec)

Fig. 3. Accelerating ramp generated by the virtual clutch.

ferent sections. Usual practices are to use TAPPI Technical Recommendations for the Normal Running Load (NRL) and Recommended Drive Capacity $(R D C)$. As a result, the power of the line-shaft drive is equivalent to the total power demanded by the sections, with some additional reserve for acceleration purposes.

In virtual line-shafting control, the "motor" rating of the virtual line-shaft is implicitly determined by the drives selected for each section which are rated by the same practices as described above, or on the basis of braking load for low-inertia sections and on stored energy for high-inertia sections. However, in virtual line-shafting control, the virtual line-shaft inertia can also play a favorable role in sectional drive ratings. Thus, the role of line-shaft inertia needs to be discussed in both physical and virtual line-shafted systems.

In the physical line-shafted system, the total line-shaft inertia as seen by any section is dictated largely by the line-shaft inertia and by pulleys (ratio) attached to the section. In physical systems, this total inertia is a very important energy storage element. The physical line-shaft machine has the inherent ability to share all of the stored energy in the machine and thus reject (lessen) the effects of section torque disturbances. From a classical machine design perspective, a large flywheel (high inertia) placed on the physical line-shaft could be used to increase the overall sectional ability to reject disturbances. However, such a flywheel would also cause high section torques to arise if disturbances occur. In a physical line-shafted system, this torque would be born by the sectional shafts and pulleys which are finite in physical torque transmission capability. This limits the "usable design range" for such a flywheel inertia on physical line-shafted systems. In contrast, if the physical line-shaft inertia is quite low, the physical line-shaft system will allow the whole system to synchronously droop in speed when large sectional disturbances occur, which effectively reduces the sectional shaft and pulley torques needed to produce such synchronization.

In the virtual line-shaft system, the virtual line-shaft inertia $J_{v}$ plays a slightly different role than in the physical system described above. The most important difference is that the virtual line-shaft inertia is not a passive energy storage element (as is the physical line-shaft flywheel). The sectional drives are actively and also "collectively" emulating the energy storage capability of the virtual line-shaft inertia and are limited in this emulation by their individual incremental power capability. The "incremental" power capability here implies the difference between the actual output of the drive and the instantaneous and short-term overload capacity of the drive.

If the virtual line-shaft system is operated within the limits of this emulation capability, then the selection of the virtual line-shaft inertia $J_{v}$ will produce similar results to the physical system which it is emulating. However, one key beneficial difference will be that the virtual line-shaft system dynamics are always well damped without the oscillatory properties which physical line-shafted systems can experience.

From a design perspective, increasing the virtual line-shaft inertia to a very large value will make the system emulate the large flywheel physical line-shaft, i.e., the system and virtual line-shaft speed droop will be minimal and the incremental sectional drive power needed to maintain synchronism will essentially be the same as that needed for today's sectional drives where no power sharing is used. In effect, an infinite value of virtual line-shaft inertia would be exactly comparable to the ex- 


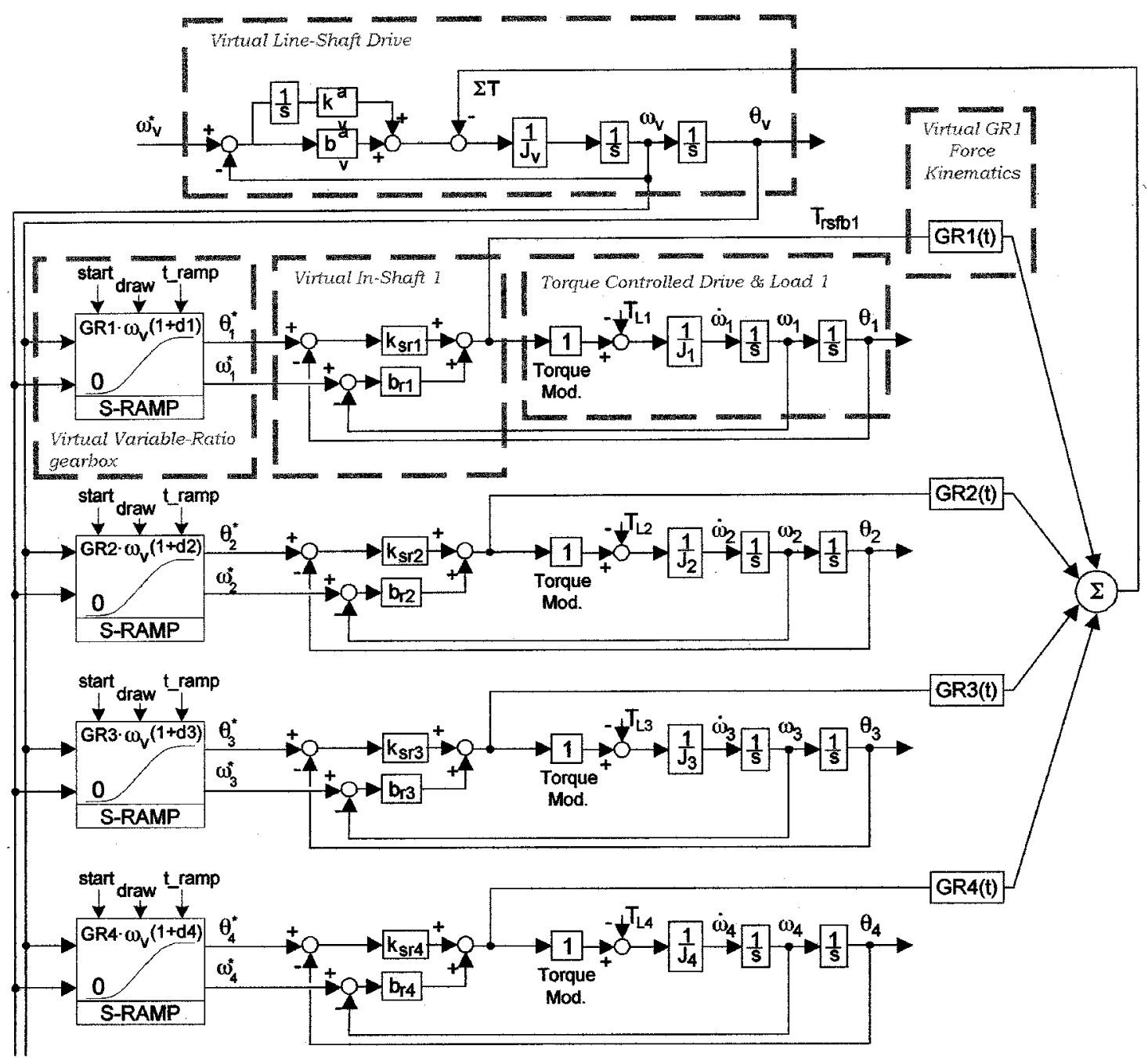

Fig. 4. Block diagram of electronic line-shafting control for a four-section system.

isting sectional drive control technology where no droop occurs in the references to the other sectional drives when one drive experiences disturbances.

On the other hand, if the virtual line-shaft inertia is decreased, then the system and virtual line-shaft speed droop will increase, but the incremental sectional drive power needed to maintain synchronism during the disturbance will be less than that needed for today's sectional drives. By emulating a slowdown of the virtual line-shaft and, thus, causing the entire machine system speed to synchronously droop slightly, the incremental sectional drive power requirements can theoretically be lessened. This is exactly why the overall machine system using a virtual lineshaft control can maintain synchronism when one of the sectional drives goes into a torque limit, i.e., the other sectional drives slow down, allowing the limited drive to catch up.

The conclusion here is that the virtual line-shaft inertia $J_{v}$ is an important design variable in virtual line-shafted systems and that it affects the incremental power requirements of the sectional drives.

The key issue in selecting the virtual line-shaft inertia is how much synchronous (and well-damped) droop is allowable. If little or no droop is allowed, then the designer increases $J_{v}$ until one of the connected drives reaches a dynamic limit for expected disturbances. This will establish the upper end of the value of virtual line-shaft inertia which can be used and maintain the desirable virtual line-shaft emulation. If some droop is allowable, then it will be better to reduce the virtual line-shaft inertia $J_{v}$ to a value which achieves this droop for expected sectional load disturbances. This can lead to a reduced incremental power rating of the sectional drives.

Thus, the value selected for $J_{v}$ will depend of the magnitude of the expected disturbances, the allowable synchronous droop, and the incremental capacity of the sectional drives to manage the disturbances without reaching their torque limits.

\section{B. Selecting the In-Shaft Parameters}

In the model of the electronic line-shaft control shown in Fig. 4, the virtual line-shaft is assumed perfectly stiff and only the stiffness and damping properties of the section in-shafts need to be considered [3]. This assumption reduces the number of parameters of the control and makes it easier to set up and commission the system.

In the physical line-shaft, the in-shaft stiffness ratings are selected according to the torque to be transferred to the mechanical sections. In the virtual in-shaft, the stiffness and damping parameters are selected to get the desired dynamic behavior, con- 
sistent with the torque capability of each section drive. It is important to note that virtual in-shaft damping can be selected to assure well damped oscillation-free operation, unlike the physical in-shaft for which adequate damping was not generally possible.

The value selected for the active stiffness parameter $K_{s r}$ will be related to the angular windup of the virtual in-shaft when transferring the torque command. A higher value of $K_{s r}$ will produce a stiffer in-shaft and lower windup. The active damping parameter $b_{s r}$ is generally much bigger than the values achievable in physical in-shafts, and is an important advantage of electronic line-shafting control. The basic tuning criteria for this parameter is based on achieving virtual in-shaft response without oscillations. After setting $K_{s r}$, the active damping $b_{s r}$ can simply be set to produce stable, nonoscillatory torque references for the current loop.

\section{Tuning the System Controllers}

Fig. 4 shows the block diagram of the electronic line-shaft control for a four-section system. The controllers of the system are the speed controller of the virtual line-shaft and the torque (current) controllers of the section drives.

The current controllers are the same used in the actual sectional drives. The tuning and bandwidth of the current controllers are usually dictated by the drive supplier and the torque control technology used for the drive. For modern field-oriented or direct-torque-controlled drives, this bandwidth is generally orders of- magnitude faster than the virtual line-shaft dynamics, and so the transients can be assumed to be negligible.

The tuning criteria of the line-shaft drive speed controller could be any of the modern algebraic methods or the more standard frequency-domain methods (state variable approach [4], optimum of magnitude [5], or the more classical Bode and loop bandwidth methods). The only caution is that the speed loop bandwidth would follow classical guidelines and thus be at least $10 \times$ slower than the torque (current) loops and would be tuned to be critically damped.

\section{SIMULATION RESUlTS}

Preliminary evaluations are done by simulation of the control system.

\section{A. Evaluation During Starting of Sections}

The starting of moderate-inertia and high-inertia sections is evaluated. The low-inertia section has a total inertia of $3 \times$ the inertia of the motor $J_{m}$, and the S-ramp is set to $12 \mathrm{~s}$. The high inertia section has a total inertia of $35 \times$ the inertia of the motor $J_{m}$ and the S-ramp is set to $17 \mathrm{~s}$. All the controller parameters are selected to get well-behaved operation. The resulting waveforms are shown in Fig. 5. Fig. 5(a) shows the virtual reference $\omega_{v}^{*}$, and the references to the sections $\omega_{1}^{*}$ and $\omega_{2}^{*}$. Fig. 5(b) and (c) show the speed and current of section 1 (moderate-inertia section), and Fig. 5(d) and (e) show the speed and current of section 2 (high-inertia section).

It can be seen that both sections are smoothly accelerated up to the reference speed without oscillations or peaks in the current or speed. (a)

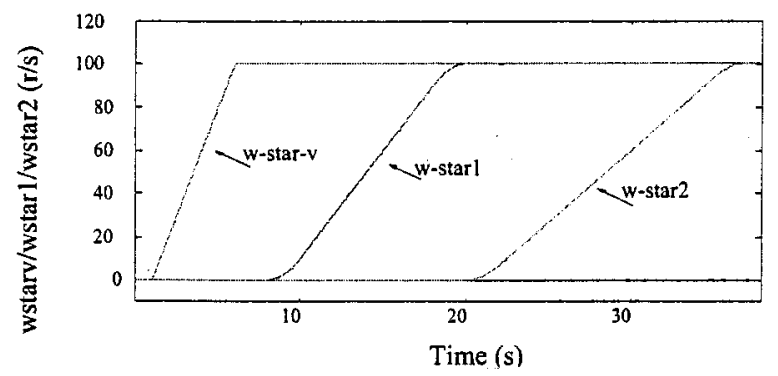

(b)

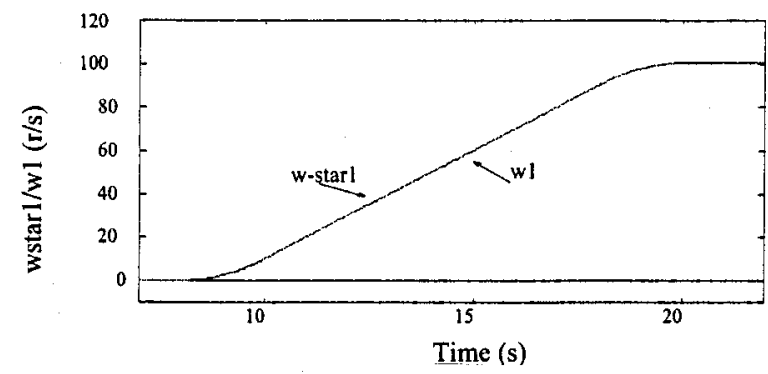

(c)

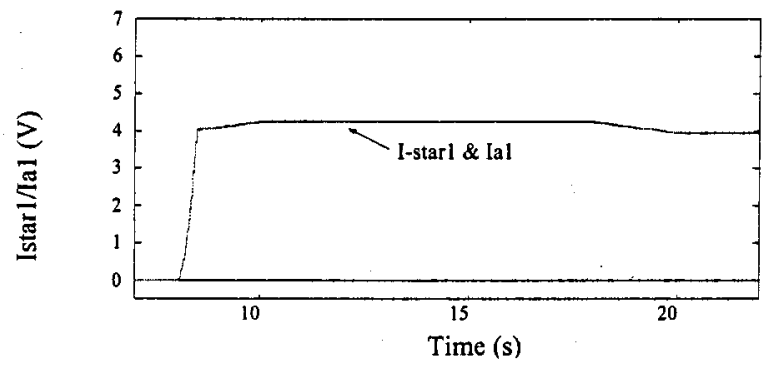

(d)

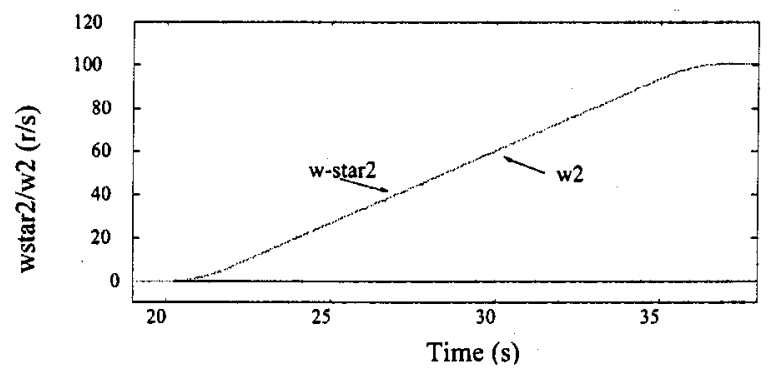

(e)

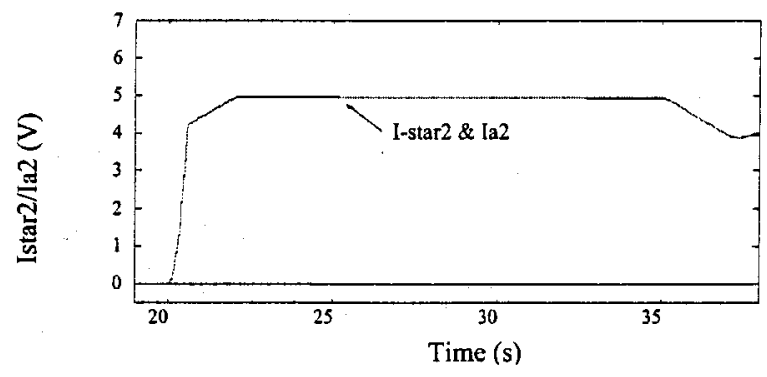

Fig. 5. Evaluation during sections starting (moderate and high inertia). (a) Velocity references. (b) Reference and velocity section 1. (c) Reference and current section 1. (d) Reference and velocity section 2. (e) Reference and current section 2 .

\section{B. Virtual Line-Shaft Inertia}

Three different values of $J_{v}$ are evaluated: low inertia $\left(J_{v o}\right)$, moderate inertia $\left(5 J_{v o}\right)$, and high inertia $\left(16.7 J_{v o}\right)$.

The lowest value of the virtual line-shaft inertia $\left(J_{v o}\right)$ was chosen equal to $1.3 \times$ the inertia of the lowest inertia section. 
(a)

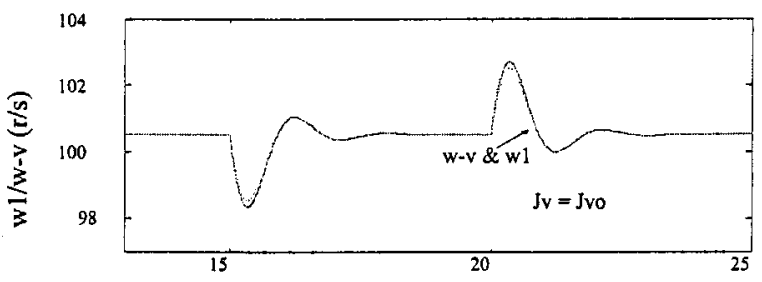

Time (s)

(b)

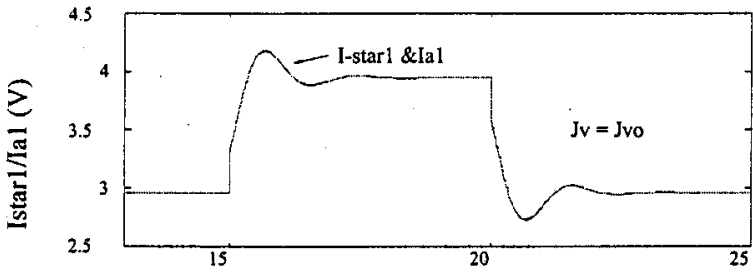

Time (s)

(c)

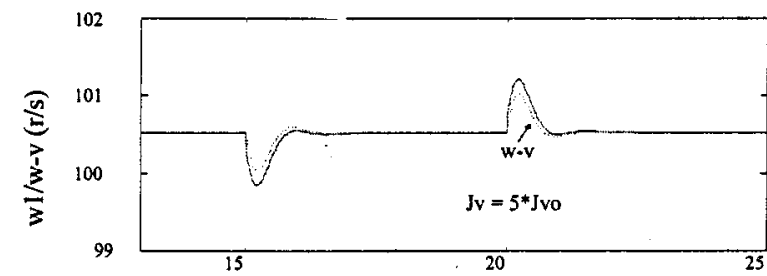

Time (s)

(d)

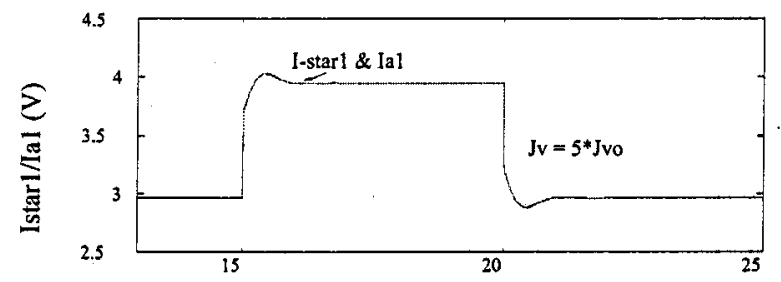

(e)

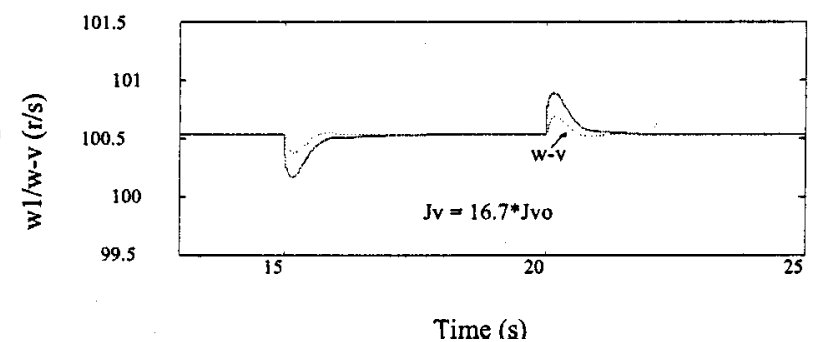

(f)

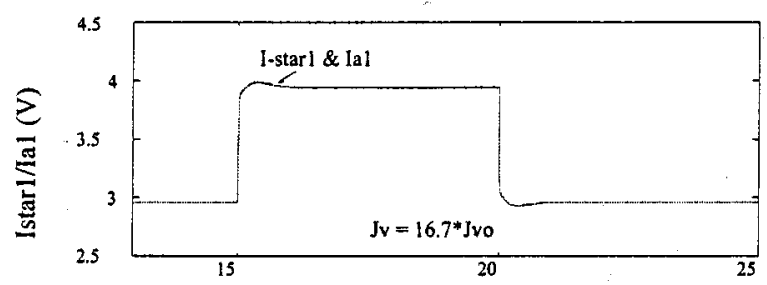

Time (s)

Fig. 6. Evaluation of virtual line-shaft inertia during 20\% step disturbance. (a) Velocity of section 1 and virtual reference $J_{v}=J_{v o}$. (b) Current reference and current, section $1, J_{v}=J_{v o}$. (c) Velocity of section 1 and virtual reference, $J_{v}=5^{*} J_{v o}$. (d) Current reference and current, section $1, J_{v}=5^{*} J_{v o}$. (e) Velocity of section 1 and virtual reference, $J_{v}=16.7^{*} J_{v o}$. (f) Current reference and current, section $1, J_{v}=16.7^{*} J_{v o}$. (a)

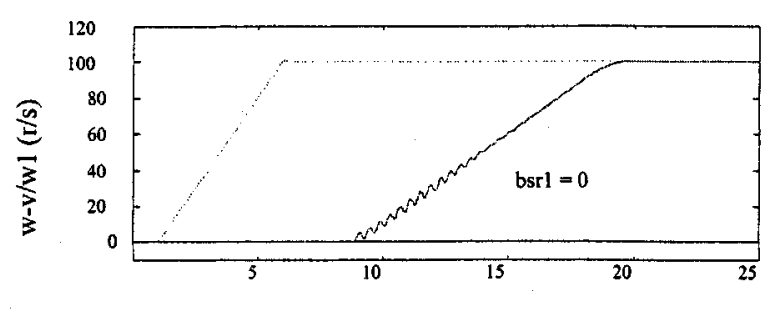

Time (s)

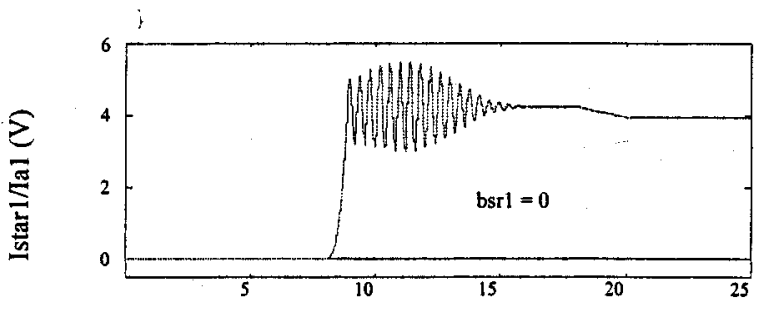

Time (s)

(c)

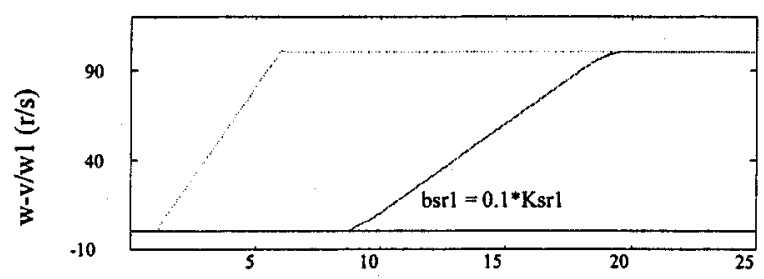

Time (s)

(d)

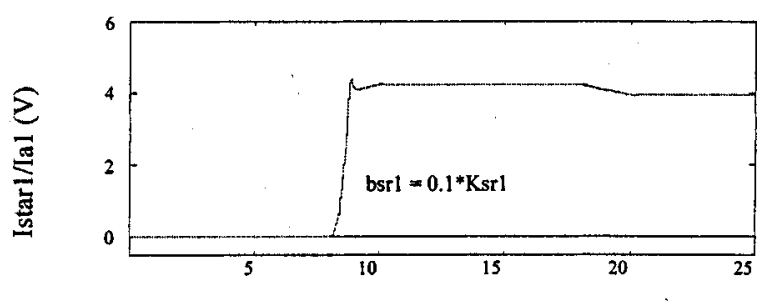

Time (s)

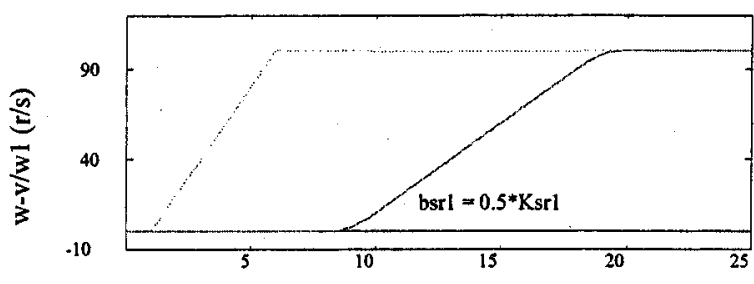

Time (s)

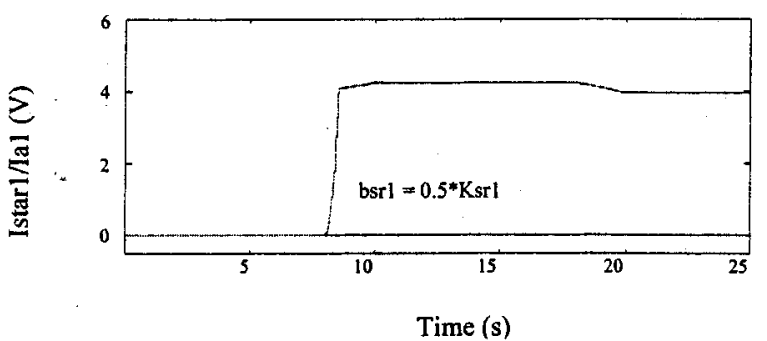

Fig. 7. Evaluation of virtual in-shaft active damping. (a) Virtual reference and velocity of section $1, b_{s r 1}=0$. (b) Current reference and current, section 1, $b_{s r 1}=0$. (c) Virtual reference and velocity of section $1, b_{s r 1}=0.1 * K_{s r 1}$. (d) Current reference and current, section $1, b_{s r 1}=0.1 * K_{s r 1}$. (e) Virtual reference and velocity of section $1, b_{s r 1}=0.5 * K_{s r 1}$. (f) Current reference and current, section $1, b_{s r 1}=0.5 * K_{s r 1}$. 


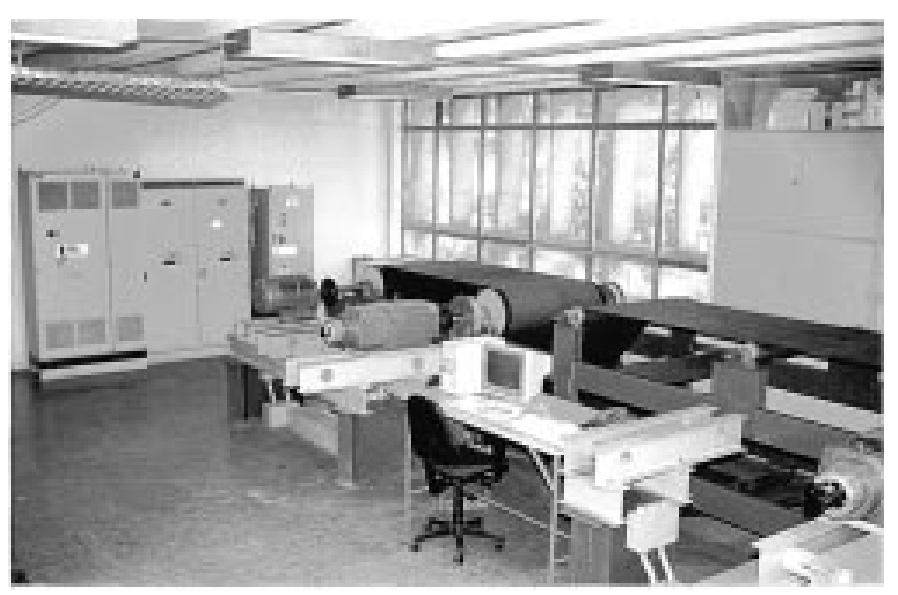

Fig. 8. Experimental setup.

Fig. 6 shows the resulting waveforms during a step load disturbance of $20 \%$ of drive rating acting on section 1 , which is assumed to be of moderate inertia.

With low $J_{v}$, the load torque reflected back to the virtual lineshaft drive produces the largest effect on the line-shaft speed $\omega_{v}$. Consequently, the speed of all sections synchronously track the line-shaft reference, allowing section 1 to handle this severe disturbance without significant deterioration of the overall synchronization to $\omega_{v}$ and to the other sections [Fig. 6(a)]. In stark contrast, using the largest $J_{v}$, the virtual speed $\omega_{v}$ remains almost constant, and section 1 is unable to maintain synchronization between $\omega_{1}$ and $\omega_{v}$ [Fig. 6(e)]. These results are illustrative of the tradeoffs when selecting low or high inertia in the virtual line-shaft drive, as explained in Section III-A.

\section{In-Shaft Parameters}

The effect of the value of virtual in-shaft stiffness $K_{s r}$ is related to the torsional windup $\Delta \Theta$ for a given torque. In this simulation, $K_{s r}$ was selected to allow about $40^{\circ}$ of torsional windup for nominal torque.

The most important parameter in order to get a well-damped response is the active damping $b_{s r}$. The evaluation shows the waveforms for three different active damping coefficients: $b_{s r}=$ $0, b_{s r}=0.1 * K_{s r}$, and $b_{s r}=0.5 * K_{s r}$. The results are plotted in Fig. 7.

When $b_{s r}=0$, the response is highly oscillatory as would be expected and, therefore, this condition is not acceptable. With the moderate value of $b_{s r}=0.1 * K_{s r}$, response is well behaved without oscillations, and just with small overshoot in the current command. With the highest value $\left(b_{s r}=0.5 * K_{s r}\right)$ the response is also well behaved and the overshoot in the current loop is removed.

\section{EXPERIMENTAL EVALUATION}

Tests were performed in the four-section laboratory testbed shown in Fig. 8. Control codes run in a digital signal processor (DSP) card, which hosts a TMS320c30 processor. Hardware also includes a 16-bit counter card, a $200-\mathrm{kHz}$ A/D-D/A card, and a data acquisition system [3]. (a)

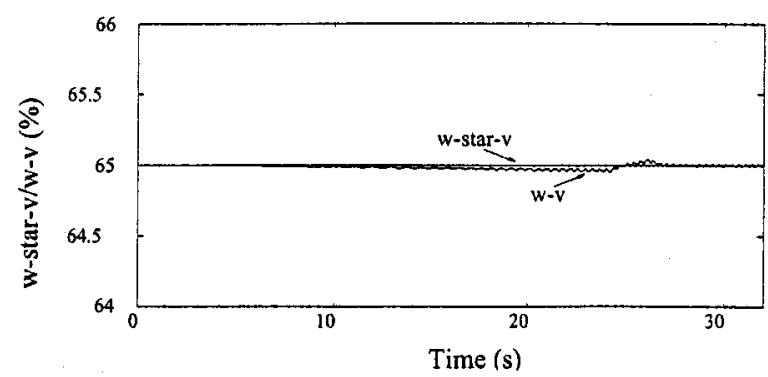

(b)

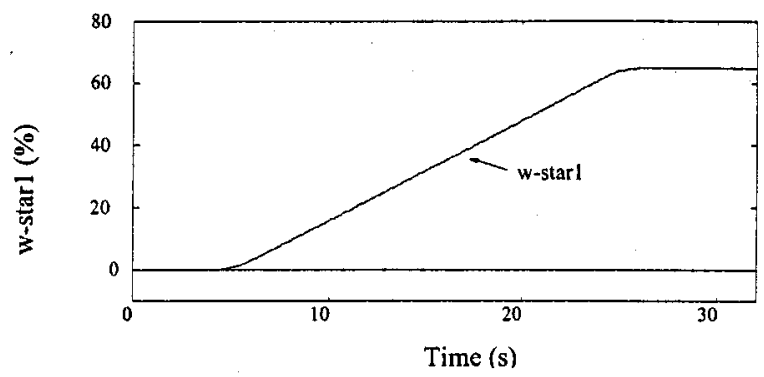

(c)

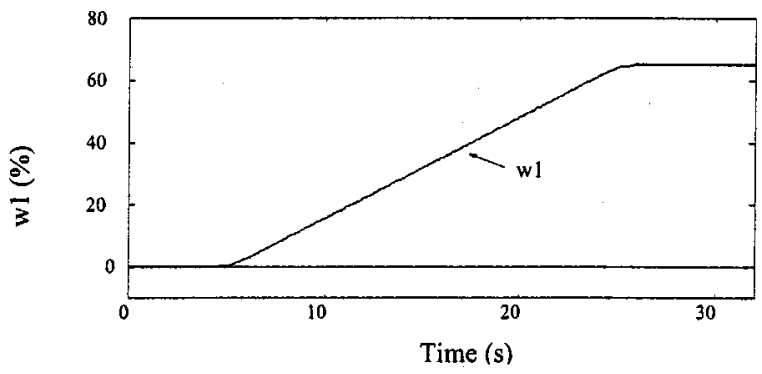

(d)

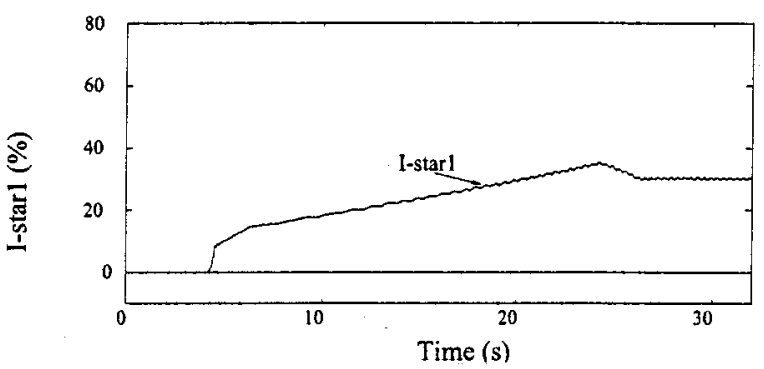

(e)

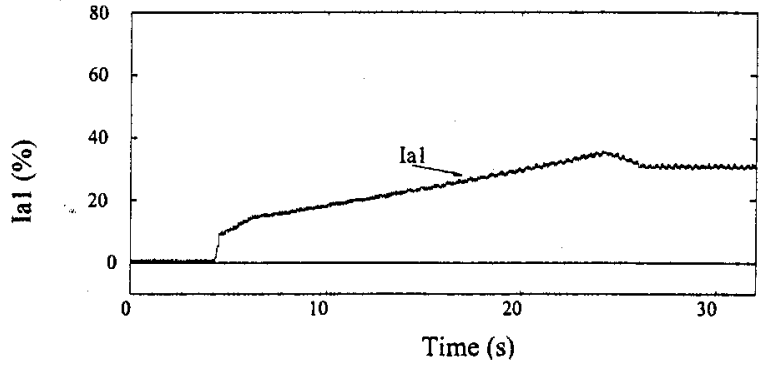

Fig. 9. Waveforms suring S-ramp starting, moderate-inertia section. (a) Master and virtual references. (b) Velocity reference, section 1. (c) Velocity of section 1. (d) Current reference, section 1. (e) Current of section 1.

\section{A. Starting of Sections}

The waveforms during an S-ramp starting of moderate- and high-inertia sections are shown in Figs. 9 and 10. Each section of the test stand has its own roll and a dc generator feeding a 
(a)

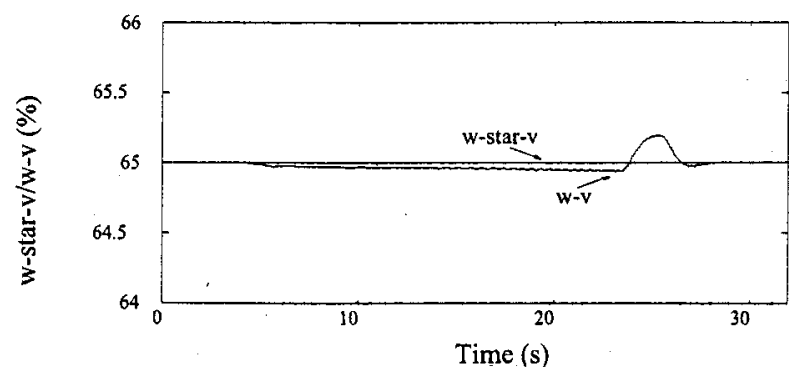

(b)

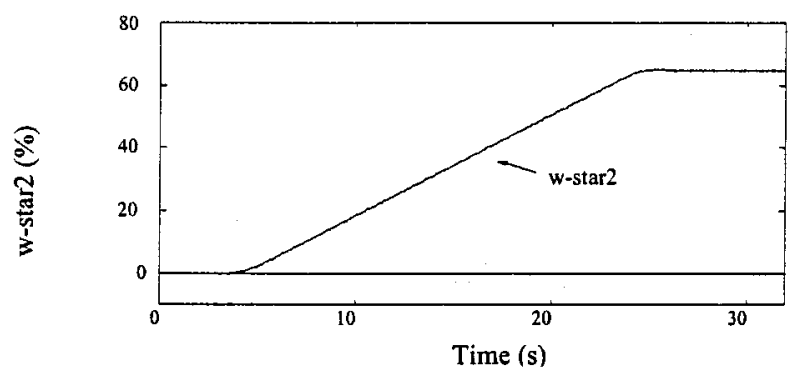

(c)

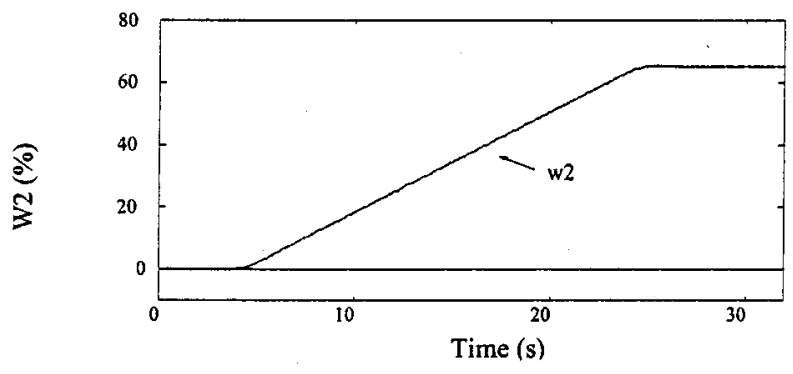

(d)

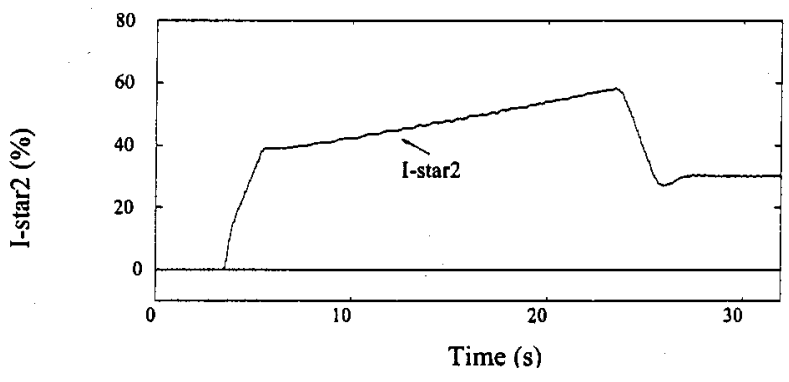

(e)

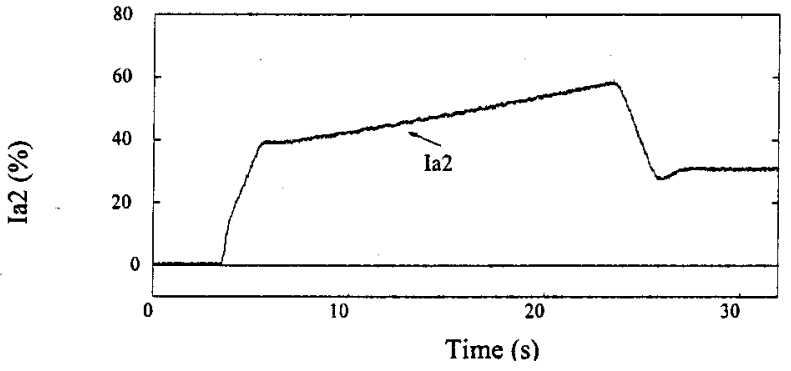

Fig. 10. Waveforms during S-ramp starting, high-inertia section 2. (a) Master and virtual references. (b) Velocity reference, section 2. (c) Velocity of section 2. (d) Current reference, section 2. (e) Current of section 2.

resistor load. This adds an increasing load torque during acceleration. In both conditions, a 20-s S-ramp is set. Recorded waveforms include virtual master reference $\omega_{v}^{*}$ and general reference to the sections $\omega_{v}$ [Figs. 9(a) and 10(a)]; the S-ramp references $\omega_{1}^{*}, \omega_{2}^{*}$ and section velocities $\omega_{1}$ and $\omega_{2}$ [Figs. 9(b) and (c) and

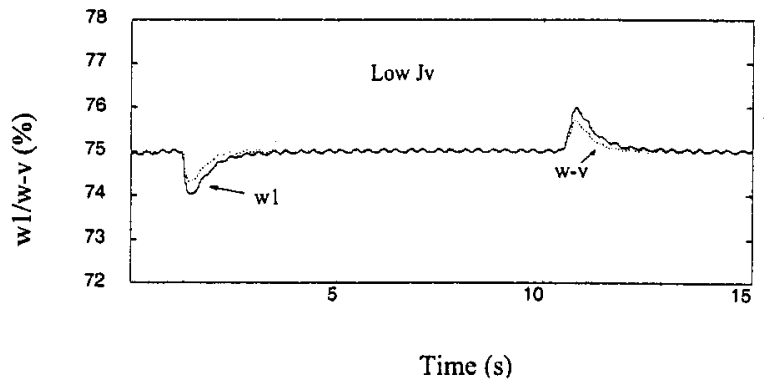

Fig. 11. Evaluation during a step load disturbance, low virtual line-shaft inertia.

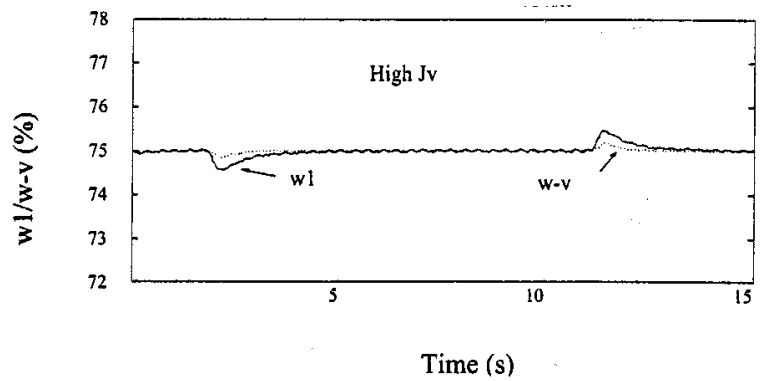

Fig. 12. Evaluation during a step load disturbance, high virtual line-shaft inertia.

10(b) and (c)]; and the current references $I_{1}^{*}$ and $I_{2}^{*}$ and stator currents $I_{a 1}$ and $I_{a 2}$ [Figs. 9(d) and (e) and 10(d) and (e)].

All these waveforms confirm that the proposed starting procedure produces smooth acceleration of the sections without undue oscillations.

\section{B. Virtual Line-Shaft Inertia}

The influence of the virtual line-shaft inertia is tested setting low- and high-inertia values in the virtual line-shaft, and evaluating the system response during a step load disturbance in section 1. Results are shown in Figs. 11 and 12. As expected, with low-inertia $J_{v}$, the virtual line-shaft allows a larger variation in synchronized response to the disturbance, which helps to maintain coordinated motion between the different sections while reducing the risk of hitting the torque limits of sections.

\section{CONCLUSIONS}

A starting procedure and tuning criteria for the parameters of an electronic line-shafting control system for paper machine drives have been proposed. Simulation and experimental evaluation in a four-section laboratory testbed confirm the appropriateness of the proposed criteria.

A virtual clutch/conical pulley assembly allows smooth acceleration of the sections, improving on the behavior of physical clutches used in classical line-shafted drives, and yields starting performance equivalent to that of actual sectional drives.

Selection of virtual line-shaft inertia $J_{v}$ depends of the magnitude of expected disturbances and the capacity available in the section drives. A low virtual inertia $J_{v}$ allows greater synchronized droop due to load disturbances, but substantially improves coordination and reduces the risk of hitting current limits. Virtual in-shaft active stiffness and damping are related to the allowed angular windup and dynamic behavior of the electronic 
line-shafting control. A high value of active stiffness $K_{s r}$ reduces the windup of sections. Active damping $b_{s r}$ is selected to assure oscillation-free response during command or load transients.

In conclusion, with the addition of the virtual clutch/conical pulley assembly, and using the proposed parameter and controller tuning criteria, the electronic line-shafting control is capable of performing all the required operating procedures.

These features, together with its capability in handing severe load disturbances and/or sustained drive saturation as was reported in [3], make the electronic line-shafting control an attractive control configuration for paper machine drives.

\section{REFERENCES}

[1] R. G. Anderson, A. J. Meyer, M. A. Valenzuela, and R. D. Lorenz, "Web machine coordinated motion control via electronic line-shafting," in Conf. Rec. IEEE-IAS Annu. Meeting, 1999, pp. 300-306.

[2] R. D. Lorenz and P. B. Schmidt, "Synchronized motion control for process automation," in Conf. Rec. IEEE-IAS Annu. Meeting, San Diego, CA, Oct. 1-5, 1989, pp. 1693-1698.

[3] M. A. Valenzuela and R. D. Lorenz, "Electronic line-shafting control for paper machine drives," in Proc. 46th Annu. IEEE Pulp and Paper Conf., Atlanta, GA, June 19-23, 2000, pp. 106-112.

[4] R. D. Lorenz, "Synthesis of state variable controllers for industrial servo drives," in Proc. Conf. Applied Motion Control, June 10-12, 1986, pp. 247-251.

[5] J. W. Umland and M. Safiuddin, "Magnitude and symmetric optimum criterion for the design of linear control systems: What is it and how does it compare with the others?," IEEE Trans. Ind. Applicat., vol. 26, pp. 489-497, May/June 1990.

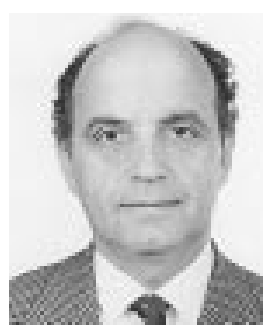

M. Aníbal Valenzuela received the electrical engineering degree and the Masters degree in electrical engineering from the University of Chile, Santiago, Chile, in 1976 and 1978, respectively.

Since 1978, has been with the Department of Electrical Engineering, University of Concepción, Concepción, Chile, where he is an Associate Professor in the area of electric machines and drives. His current research interests include motion control of multidrive systems, sensorless control of ac drives, and thermal evaluation of induction motors.

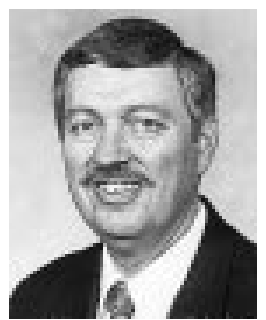

Robert D. Lorenz (S'83-M'84-SM'91-F'98) ) received the B.S., M.S., and Ph.D. degrees from the University of Wisconsin, Madison, and the M.B.A. degree from the University of Rochester, Rochester, NY.

Since 1984, he has been a member of the faculty of the University of Wisconsin, Madison, where he is the Mead Witter Foundation Consolidated Papers Professor of Controls Engineering in both the Department of Mechanical Engineering and the Department of Electrical and Computer Engineering. He is Co-Director of the Wisconsin Electric Machines and Power Electronics Consortium, which celebrated its 20th anniversary in 2001. It is the largest industrial research consortium on motor drives in the world. He is also the thrust leader for control and sensor integration in the Center for Power Electronic Systems, an NSF Engineering Research Center (ERC) which is a joint ERC with Virgina Polytechnic Institute and State University, Rensselaer Polytechnic Institute, University of Puerto Rico-Mayaguez, and North Carolina A\&T. From 1972 to 1982, he was a member of the research staff at the Gleason Works, Rochester, NY, working principally on high-performance drives and synchronized motion control. He was a Visiting Research Professor in the Electrical Drives Group, Catholic University of Leuven, Leuven, Belgium, in the summer of 1989 and in the Power Electronics and Electrical Drives Institute, Technical University of Aachen, Aachen, Germany, in the summers of 1987, 1991, 1995, 1997, and 1999, where he also was the SEW Eurodrive Guest Professor from September 1, 2000 until July 7, 2001. In 1969-1970, he conducted Master thesis research in adaptive control of machine tools at the Technical University of Aachen. His current research interests include sensorless electromagnetic motor/actuator technologies, real-time signal processing and estimation techniques, precision multiaxis motion control, and ac/dc drive and high-precision machine control technologies. He has authored more than 160 published technical papers and is the holder of more than 15 patents, with five more pending.

Dr. Lorenz was the IEEE Industry Applications Society (IAS) President for 2001, a Distinguished Lecturer of the IAS for 2000/2001, immediate past Chair of the IAS Awards Department, and past Chairman of the IAS Industrial Drives Committee, and is a member of the IAS Industrial Drives, Electric Machines, Industrial Power Converter, and Industrial Automation and Control Committees. $\mathrm{He}$ is also the current Chair of the Periodicals Committee for the IEEE Technical Activities Board. He is a member of the IEEE Sensor Council AdCom and the IEEE Neural Network AdCom. He is a Registered Professional Engineer in the States of New York and Wisconsin. He is also a member of the American Society of Mechanical Engineers, Instrument Society of America, and Society of PhotoOptical Instrumentation Engineers. He has won 13 prize paper awards. 